\title{
Impact of immunosuppressant therapy on early recurrence of hepatocellular carcinoma after liver transplantation
}

\author{
Ju-Yeun Lee ${ }^{1,2}$, Yul Hee Kim³ ${ }^{3}$ Nam-Joon Yi", Hyang Sook Kim', Hye Suk Lee', Byung Koo Lee ${ }^{3}$, Hyeyoung Kim ${ }^{4}$, Young \\ Rok Choi ${ }^{4}$, Geun Hong ${ }^{5}$, Kwang-Woong Lee ${ }^{4}$, and Kyung-Suk Suh ${ }^{4}$ \\ 'Department of Pharmacy, Seoul National University Hospital, Seoul National University College of Medicine, Seoul; ${ }^{2}$ College of Phar- \\ macy, Hanyang University, Gyeonggi-do; ${ }^{3}$ Ewha Graduate School of Clinical Health Sciences, Ewha Women's University, Seoul, ${ }^{4}$ Depart- \\ ment of Surgery, Seoul National University College of Medicine, Seoul; ${ }^{5}$ Department of Surgery, Ewha Women's University College of \\ Medicine, Seoul, Korea
}

Background/Aims: The most commonly used immunosuppressant therapy after liver transplantation (LT) is a combination of tacrolimus and steroid. Basiliximab induction has recently been introduced; however, the most appropriate immunosuppression for hepatocellular carcinoma (HCC) patients after LT is still debated.

Methods: Ninety-three LT recipients with HCC who took tacrolimus and steroids as major immunosuppressants were included. Induction with basiliximab was implemented in 43 patients (46.2\%). Mycophenolate mofetil (MMF) was added to reduce the tacrolimus dosage $(\mathrm{n}=28,30.1 \%)$. The 1-year tacrolimus exposure level was $7.2 \pm 1.3 \mathrm{ng} / \mathrm{mL}$ (mean $\pm S D$ ).

Results: The 1- and 3-year recurrence rates of HCC were $12.9 \%$ and 19.4\%, respectively. Tacrolimus exposure, cumulative steroid dosages, and MMF dosages had no impact on HCC recurrence. Induction therapy with basiliximab, high alpha fetoprotein (AFP; $>400 \mathrm{ng} / \mathrm{mL}$ ) and protein induced by vitamin $\mathrm{K}$ absence/antagonist-II (PIVKA-Il; $>100 \mathrm{mAU} / \mathrm{mL}$ ) levels, and microvascular invasion were significant risk factors for 1 -year recurrence $(P<0.05)$. High AFP and PIVKA-II levels, and positive ${ }^{18}$ fluoro-2-deoxy-d-glucose positron-emission tomography findings were significantly associated with 3-year recurrence $(P<0.05)$.

Conclusions: Induction therapy with basiliximab, a strong immunosuppressant, may have a negative impact with respect to early HCC recurrence (i.e., within 1 year) in high-risk patients. (Clin Mol Hepatol 2014;20:192-203)

Keywords: Immunosuppression; Basiliximab; Microvascular invasion; PIVKA-II; AFP; ${ }^{18}$ F-PET scan

\section{INTRODUCTION}

Hepatocellular carcinoma (HCC) is the seventh most common cancer and the third leading cause of cancer mortality worldwide.' Liver transplantation (LT) has become the treatment of choice for the early stage of unresectable HCC patients because it offers complete tumor excision along with the removal of the carcino- genic liver. Unfortunately, tumor recurrence after LT still remains the main cause of death for HCC patients, and the incidence of recurrence is reported to be between $15 \%$ and $20 \%{ }^{2}$ Tumor progression is more rapid and aggressive in immunosuppressed patients following LT. The degree of the immunosuppression negatively affects the post-LT recurrence of HCC as well as the long-term survival of such patients. ${ }^{3}$

\footnotetext{
Abbreviations:

18-FDG PET, 18-fluoro-2-deoxy-D-glucose positron emission tomography; AFP, alpha-fetoprotein; AUC, area under the curve; Cl, confidence interval; $\mathrm{CT}$, computed tomography; DDLT, deceased donor liver transplantation; HCC, hepatocellular carcinoma; HR, hazard ratio; LDLT, living donor liver transplantation; LT, liver transplantation; MELD, model for end-stage liver disease; MMF, Mycophenolate mofetil; MVI, microvascular invasion; PIVKA-II, protein induced by vitamin Kabsence-II

\section{Corresponding author : Nam-Joon Yi}

Department of Surgery, Seoul National University Hospital, 101 Daehakro, Jongno-gu, Seoul 110-744, Korea

Tel. +82-2-2072-2990, Fax. +82-2-766-3975

E-mail; gsleenj@hanmail.net 
Attempts to identify clinical variables that influence tumor recurrence have resulted in improved selection criteria for patients with favorable HCC. Tumor size, number, differentiation, vascular invasion, and the serum alpha-fetoprotein (AFP) levels are potential markers for recurrence. ${ }^{4-6}$ Research into the relation between immunosuppressive regimens and tumor recurrence are ongoing in animal models and a few clinical studies. Calcineurin inhibitors and steroids dose-dependently increase the risk of HCC recurrence, although these are main immunosuppressants in LT recipients. $^{7-9}$ Sirolimus has an anti-proliferative and anti-tumor effect, ${ }^{10}$ but is not approved for use in LT." The choice of immunosuppressive regimen for decreasing tumor recurrence risk is still a matter of debate.

Most research of immunosuppressants and HCC recurrence were analyzed in a deceased donor LT (DDLTs) setting. The immunosuppressant requirements are usually lower in recipients of living donor LTs (LDLTS) than in recipients of DDLTs. Recipients with hepatitis B related liver disease showed lower rejection rates compared to the other disease categories. ${ }^{12}$ Our center is a large volume LDLT center and has mostly adult recipients (around 80\%) with hepatitis B related liver disease..$^{13}$ In this patient population, our center usually follows our immunosuppressant guidelines for $\mathrm{HCC}$ recipients consisting of low levels of tacrolimus (5 to $8 \mathrm{ng} / \mathrm{mL}$ during the first year and $5 \mathrm{ng} / \mathrm{mL}$ thereafter) and steroids which are usually tapered down within 6 months. Basiliximab, a chimeric monoclonal antibody of the interleukin-2 receptor antagonist, has been shown to be useful as induction therapy in the setting of pre-transplant renal dysfunction because it allows minimization and delayed introduction of calcineurin inhibitors after LT. ${ }^{14}$ An induction therapy of basiliximab and addition of mycophenolate mofetil (MMF) has recently come into use in critically ill patients with encephalopathy or poor renal function, who were saved by delaying tacrolimus during the immediate post-LT period.

The objective of this study was to retrospectively investigate the effect(s) of different immunosuppressant exposures on HCC recurrence after LT-along with the many clinical, pathological, and histological factors-in a single large volume LDLT center.

\section{METHODS}

\section{Patients}

Between January 2005 and September 2009, 108 adult patients with HCC who received tacrolimus and steroids as the main immunosuppressant after LT at Seoul National University Hospital were evaluated. Of these patients, 15 patients (13.9\%) were excluded: three patients with a history of other organ malignancy, four patients with metastasis in other organs at the time of the LT, and eight patients who changed main immunosuppressants within one year. Therefore, 93 patients were included as study subjects. Electronic medical records for these 93 patients were reviewed and the data collected

Post-transplant surveillance for HCC recurrence included serum AFP, PIVKA-II level measurements during each outpatient clinic visit and abdominal CT scans for 1, 6, 12, 18, 24, and 36 months after transplantation. Chest CT scans and bone scans were conducted if HCC recurrence was suspected in any clinical or serological findings. The patients were evaluated via enhanced 3D-spiral CT scans, enhanced dynamic magnetic resonance imaging, and 18-fluoro-2-deoxy-D-glucose positron emission tomography (18FFDG PET) scans.

No neoadjuvant or adjuvant systemic chemotherapy was administered to the patients in this study.

All aspects of the retrospective study protocol were approved by our local Institutional Review Board (H-1110-100-382).

\section{Clinical and pathological factors for HCC}

In pre-transplant data, the recipient gender, age at the time of transplant, donor type, underlying liver disease, Child-Pugh class, medical Model for End-Stage Liver Disease (MELD) score without additional score for HCC, AFP level, protein induced by vitamin $\mathrm{K}$ absence-II (PIVKA-II) level, positive PET scans, and the pre-transplant treatment were reviewed. The pathological status of the explanted liver was reviewed; the number of tumors, tumor size, tumor differentiation, microvascular invasion (MVI), serosal invasion, intrahepatic metastasis, tumor stage, and Milan criteria fulfillment were documented. A positive PET scan finding was defined as an observed greater uptake of 18F-FDG in a primary HCC lesion than in a background liver. ${ }^{15}$ Tumor differentiation was classified in four grades according to Edmondson-Steiner (ES) criteria, and tumor stages were determined by pTNM classification (AJCC $7^{\text {th }}$ edition). For post-transplant data, the presence of histologically proven acute rejection (Banff score) episodes and their treatment were analyzed.

\section{Immunosuppressive therapy}

Tacrolimus dosage was adjusted to the target trough blood level 
of 8 to $10 \mathrm{ng} / \mathrm{mL}$ for the first two weeks, followed by 5 to $8 \mathrm{ng} / \mathrm{mL}$ during the first year, and $5 \mathrm{ng} / \mathrm{mL}$ thereafter. Whole blood trough levels of tacrolimus were measured with a microparticle enzyme immunoassay (Tacrolimus, Abbott Laboratories, Abbott Park, IL). To assess the impact of tacrolimus exposure on tumor recurrence, average tacrolimus exposure was defined as the area under the curve (AUC) of tacrolimus levels plotted against time courses until the time to recurrence or one month, 3 month, 6 month, and one year after transplantation divided by the time of exposure to the tacrolimus. ${ }^{6}$ Tacrolimus blood levels at 3, 7, and 14 days, and 1, 3, 6,9 , and 12 months were used for calculating the AUC. The AUC was calculated by using the trapezoidal rule.

Methylprednisolone was initially given intravenously for the first 6 days after surgery and then changed to oral prednisolone. Steroids were progressively tapered to discontinuation by 3 to 6 months after LT. MMF was added in 28 patients (30.1\%). The cumulative dosages of steroids and MMF during the first year were calculated. Basiliximab was introduced in 43 recipients (46.2\%).

\section{Statistical Analysis}

Continuous variables are reported as the median (range) or mean \pm the standard deviation, and differences in subgroups were compared using Student's t-test. Categorical variables were reported in a number of cases, and prevalence and differences in subgroups were compared using a chi-square test or Fisher's exact test with Yates correction. The optimal cut-off of tacrolimus exposure was obtained using receiver operating characteristic (ROC) curve. To analyze the impact of tumor recurrence by individual variables, a univariate Cox regression model was used. To identify independent variables that affect tumor recurrence, a stepwise multivariate Cox proportional hazard model was applied. Recurrence rates were calculated using survival analysis based on the Kaplan-Meier method, and a log-rank test was used to compare each subgroup. Null hypotheses of no difference were rejected if $P$-values were less than .05 . Statistical analysis was performed using the SPSS Version 18.0 for Windows package (SPSS, Chicago, IL).

\section{RESULTS}

\section{The demographic data and tumor characteristics}

The demographic data and tumor characteristics of the study populations are described in Table 1. The most common original liver disease was related to hepatitis B virus (83.9\%). LDLT was performed in $90.3 \%(n=84)$ of the patients. The mean medical MELD score without additional point for HCC was $15.0 \pm 7.23$ at the time of transplantation. The patients with serum AFP level of more than $400 \mathrm{ng} / \mathrm{mL}$ were $16.1 \%(n=15)$ and the patients with PIVKA-II level of more than $100 \mathrm{mAU} / \mathrm{mL}$ were $22.6 \%(n=21)$. Thirty-three percent $(n=31)$ had undergone some form of pretransplant treatment against HCC, i.e., trans-arterial chemoembolization, percutaneous ethanol injection therapy, or radiofrequency ablation including multiplex treatment. Based on the results of imaging studies, $75.3 \%(n=70)$ of the patients met radiologic Milan criteria. The PET scans were positive in $18.1 \%(n=17)$.

Pathologic data showed that maximum tumor diameter of more than $5 \mathrm{~cm}$ was present in $7.5 \%(n=7)$ of the patients, and more than three tumors were present in $29.0 \%(n=27)$. A total of $49.5 \%(n=46)$ patients showed grades 3 and 4 differentiation based on a worst of ES score system. MVI was noted in $6.5 \%$ $(n=6)$ of the patients, serosal invasion in $12.9 \%(n=12)$, and intrahepatic metastasis in $14.0 \%(n=13)$ of the patients. The pathologic tumor stage was pT1 in $53.8 \%(n=50)$, and more than pT2 in $46.2 \%(n=43)$.

\section{Survival Outcomes}

The mean follow-up period for the entire study population was $923 \pm 344$ (21-1095) days. Recurrence of HCC was observed in 12 patients $(12.9 \%)$ within one year after LT and in 18 patients (19.4\%) within 3 year of LT. In other words, the overall one- and three-year recurrence free rate were $87.1 \%$ and $80.6 \%$, respectively (Fig.1). Recurrence was diagnosed between 71 and 771 days after LT (median 333 days). The sites of recurrence were the graft liver $(n=4)$, lungs $(n=3)$, bones $(n=3)$, peritoneal seeding $(n=3)$, celiac lymph node $(n=1)$, adrenal gland $(n=1)$, and multiple organs $(n=4)$. Two patients died without HCC recurrence and are regarded as censored cases.

\section{Analysis of Immunosuppressant}

The average one month tacrolimus exposure was $9.32 \pm 2.04$ $\mathrm{ng} / \mathrm{mL}, 3$ month was $8.80 \pm 1.74 \mathrm{ng} / \mathrm{mL}$, 6 month was $8.10 \pm 1.47$ $\mathrm{ng} / \mathrm{mL}$, and one year was $7.23 \pm 1.31 \mathrm{ng} / \mathrm{mL}$. There were no significant differences between the patients with and without 1-year recurrence in tacrolimus average exposure during the entire course of this study (Table 2). However, one year tacrolimus average exposure in patients with 3-year recurrence $(7.97 \pm 1.35 \mathrm{ng} / \mathrm{mL})$ was 
Table 1. Characteristics of the patients and their tumors

\begin{tabular}{|c|c|}
\hline Variables & \\
\hline \multicolumn{2}{|l|}{ Clinical information } \\
\hline Age (median years, range) & $53(32-65)$ \\
\hline Sex (male/female), n (\%) & $75(80.6) / 18(19.4)$ \\
\hline Donor type (living/ deceased), n (\%) & $84(90.3) / 9(9.7)$ \\
\hline \multicolumn{2}{|l|}{ Etiology } \\
\hline Non-viral/HBV/HCV/HBV + HCV, n (\%) & $4(4.3) / 78(83.9) / 9(9.7) / 2(2.1)$ \\
\hline Medical MELD score (mean \pm SD) & $15.30 \pm 7.23$ \\
\hline \multicolumn{2}{|l|}{ Child-Pugh class } \\
\hline Class A/Class B/Class C, n (\%) & $22(23.7) / 38(40.8) / 33(35.5)$ \\
\hline $\operatorname{AFP}(n g / m L)(\leq 400 />400), n(\%)$ & $78(83.9) / 15(16.1)$ \\
\hline PIVKA-II level (mAU/mL) $\leq 100 />100, n(\%)$ & $72(77.4) / 21(22.6)$ \\
\hline PET (positive/negative), n (\%) & $17(18.1) / 76(81.9)$ \\
\hline Preoperative treatment (yes/no), n (\%) & $31(33.3) / 62(66.7)$ \\
\hline Milan criteria (fulfilled/unfulfilled), n (\%) & $70(75.3) / 23(24.7)$ \\
\hline \multicolumn{2}{|l|}{ Pathologic data of HCC } \\
\hline Maximum tumor diameter $(\leq 5 \mathrm{~cm} />5 \mathrm{~cm}), \mathrm{n}(\%)$ & $86(92.5) / 7(7.5)$ \\
\hline Number of tumors $(<3 / \geq 3), n(\%)$ & $66(71.0) / 27(29.0)$ \\
\hline \multicolumn{2}{|l|}{ Tumor differentiation, worst (ES grade) } \\
\hline Grade 1-2/Grade 3-4, n (\%) & $47(50.5) / 46(49.5)$ \\
\hline Microvascular invasion (yes/no), n (\%) & $6(6.5) / 87(93.5)$ \\
\hline Serosal invasion (yes/no), n (\%) & $12(12.9) / 81(87.1)$ \\
\hline Intrahepatic metastasis (yes/no/unknown) & $13(14.0) / 78(83.9) / 2(2.1)$ \\
\hline Pathologic tumor stage (pT1/pT2-pT3) & $50(53.8) / 43(46.2)$ \\
\hline \multicolumn{2}{|l|}{ Immunosuppression } \\
\hline Rejection episode (yes/no), n (\%) & $16(17.2) / 77(82.8)$ \\
\hline Use of basiliximab (yes/no), n (\%) & $43(46.24) / 50(53.76)$ \\
\hline Tacrolimus + MMF + steroids/tacrolimus + steroids, n (\%) & $28(30.1) / 65(69.9)$ \\
\hline
\end{tabular}

higher than patients without recurrence $(7.05 \pm 1.24 \mathrm{ng} / \mathrm{mL})$ $(P=0.015)$. A cut-off point of one year average exposure to tacrolimus $\geq 7.5 \mathrm{ng} / \mathrm{mL}$ was identified by ROC analysis, with an AUC of 0.701 (95\% Cl $0.562-0.840)$, sensitivity of $72.2 \%$ and specificity of $64.9 \%$. There was a high proportion of patients with high exposure to tacrolimus ( $\geq 7.5 \mathrm{ng} / \mathrm{mL}$ ) in 3-year recurrence group (72.2\%) than non-recurrence group (35.2\%) ( $P=0.007$ ) (Table 3).

The one year cumulative steroid dosage was 3,940.10 \pm 1417.16 $(2,060-14,296) \mathrm{mg}$. There were no differences in cumulative steroid dosage between the patients with one-year HCC recurrence $(3,620.9 \pm 538.6 \mathrm{mg})$ and the patients without recurrence
$(3,788.2 \pm 749.2 \mathrm{mg})(P=0.459)$. The cumulative steroid dosage was not different between the patients with 3 -year HCC recurrence $(3,575.7 \pm 480.7 \mathrm{mg})$ and the patients without recurrence $(3,813.0 \pm 767.8 \mathrm{mg})(P=0.211)$.

The one year cumulative MMF dosage was 201.6 \pm 11.9 (120.0412.0) g. The cumulative MMF dosage was not different between the patients with one-year HCC recurrence $(141.0 \pm 86.5 \mathrm{~g})$ and the patients without recurrence $(232.0 \pm 120.1 \mathrm{~g})(P=0.061)$. The cumulative MMF dosage was also not different between patients with 3-year HCC recurrence $(177.3 \pm 124.3 \mathrm{~g})$ and patients without recurrence $(224.2 \pm 117.3 \mathrm{~g})(P=0.374)$. 


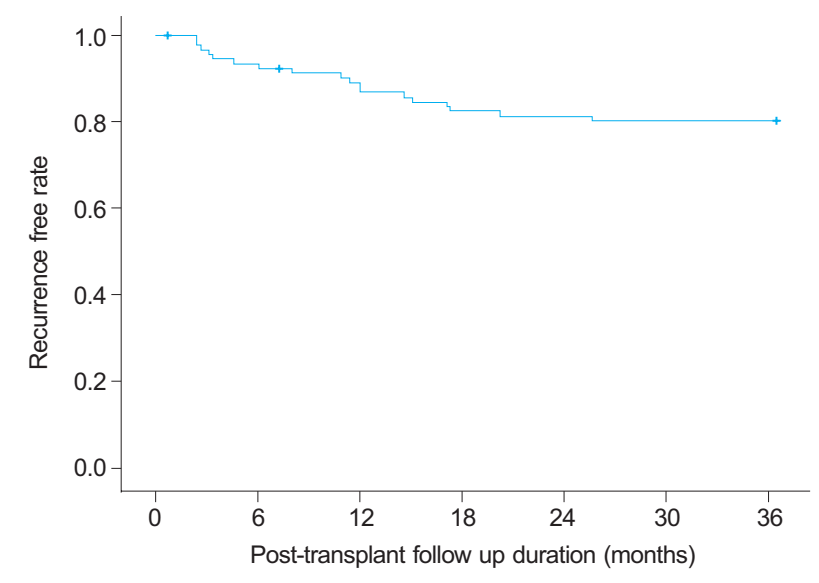

$\begin{array}{llllllll}\text { No. at risk } & 93 & 86 & 79 & 75 & 74 & 73 & 73\end{array}$

Figure 1. Recurrence-free interval curve after liver transplantation in patients with HCC. The 1- and 3-year recurrence-free rates were $87.1 \%$ and $80.6 \%$, respectively.

The basiliximab induction therapy was more frequently used in one-year recurrence group than non-recurrence group (75.0\% vs. $42.0 \%, P=0.032$ ); however the frequency of basiliximab induction therapy was not significantly different between patients with and without 3-year recurrence (61.1\% vs. $42.7 \%, P=0.159)$

\section{Factors affecting the one-year recurrence out- comes}

With regard to non-pharmacological factors, pre-transplant AFP levels over $400 \mathrm{ng} / \mathrm{mL}(P<0.001)$, PIVKA-II levels over $100 \mathrm{mAU} / \mathrm{mL}$ $(P=0.002)$, tumor sizes over $5 \mathrm{~cm}(P=0.018)$, tumor differentiation of G3-G4 ( $P=0.025)$, PET positive $(P<0.001)$, MVI $(P<0.001)$, serosal invasion $(P=0.026)$, pathologic tumor stage pT2-pT3 $(P=0.016)$, and unfulfilled radiologic Milan criteria $(P=0.005)$ were variables that significantly affected one-year $\mathrm{HCC}$ recurrence by univariate analysis. With regard to pharmacological factors, and use of induction therapy with basiliximab $(P=0.043)$ affected one year HCC recurrence by univariate analysis (Table 4).

In multivariate analysis, the variates found significant ( $p$-value $<0.05$ ) by univariate analysis except tumor size which is associated with both Milan criteria and tumor stage were included. In multivariate Cox regression analysis adjusted for age, gender, and medical MELD score, AFP levels (HR: 87.24, 95\% Cl: 9.25-822.64, $P<0.001)$, induction therapy with basiliximab (HR: 19.73, Cl: 2.78134.00, $P=0.003)$, PIVKA-Il levels (HR: 11.42, Cl: 2.04-63.83, $P=0.006)$, and MVI (HR: 6.06, Cl: 1.23-29.91, $P=0.0271)$ (Fig.2) were independently and significantly associated with one-year recurrence outcome (Table 4).

\section{Factors affecting the 3-year recurrence out- come}

With regard to non-pharmacological factors, pre-transplant AFP levels over $400 \mathrm{ng} / \mathrm{mL}(P<0.001)$, PIVKA-II levels over $100 \mathrm{mAU} /$ $\mathrm{mL}(P<0.001)$, tumor sizes over $5 \mathrm{~cm}(P=0.002)$, tumor differentiation of G3-G4 $(P=0.002)$, PET positive $(P<0.001)$, MVI $(P=0.001)$, serosal invasion $(P<0.001)$, TNM stage pT2-pT3 $(P=0.002)$, and unfulfilled radiologic Milan criteria $(P=0.004)$, number of tumors more than $3(P=0.027)$, and intrahepatic metastasis $(P=0.022)$ were variables that significantly affected $\mathrm{HCC}$ recurrence by univariate analysis. With regard to pharmacological factors, one year average tacrolimus exposure more than $7.5 \mathrm{ng} / \mathrm{mL}(P=0.008)$ affected 3-year HCC recurrence by univariate analysis (Table 5$)$.

In multivariate analysis, the variates found significant $(P$-value $<0.05$ ) except tumor size and tumor number which are associated with both Milan criteria and tumor stage were included. By a multivariate Cox regression analysis adjusted for age, gender, and medical MELD score, AFP levels (HR: 15.99, 95\% Cl: 4.42-57.88, $P<0.001)$, PIVKA-II levels (HR: 4.25, Cl: 1.24-214.56, $P=0.021$ ), and PET positive (HR: 9.49, $\mathrm{Cl}: 2.47-36.47, P=0.001$ ) were independently and significantly associated with three-year recurrence outcome (Table 5).

\section{DISCUSSION}

Immunosuppressive therapy after LT has changed over the last 10 years. Minimization of calcineurin inhibitor exposure and steroid therapy combination with MMF has become widely adopted. Although postoperative immunosuppression can accelerate tumor growth, there are only a few studies that investigate the possible influence of different immunosuppressive agents on HCC recurrence after liver transplantation. ${ }^{4,6}$ In contrast, it is widely accepted that the pathologic status of HCC is closely related to the risk of $\mathrm{HCC}$ recurrence, and patient survival has improved by establishing Milan criteria for selecting transplant candidates. ${ }^{5,16-20}$ In this regard, we focused on immunosuppressive agents and the early recurrence (within one year) of HCC because exposure to immunosuppressants was maximal during this period, the HCC which were recurred within one year might have aggressive nature, and the late recurrence of $\mathrm{HCC}$ might be related to many complex factors. In our study, basiliximab induction therapy was the independent risk factor of early recurrence within one-year. This could be explained by the effect of strong immunosuppression on the early 
Table 2. Comparison of the characteristics of patients with/without 1-year recurrence

\begin{tabular}{|c|c|c|c|}
\hline Variables & Recurrence group ( $n=12$ ) & Non- Recurrence group $(n=81)$ & $P$-value \\
\hline Age $($ mean $\pm S D)$ & $56.6 \pm 4.23$ & $52.6 \pm 7.06$ & 0.013 \\
\hline Sex (Male/Female), n (\%) & $10(83.3) / 2(16.7)$ & $65(80.2) / 16(19.8)$ & 0.801 \\
\hline Donor type (Living/Deceased), n (\%) & $11(91.7) / 1(8.3)$ & $73(90.1) / 8(9.9)$ & 0.866 \\
\hline \multicolumn{4}{|l|}{ Etiology } \\
\hline Non-viral/HBV, n (\%)/HCV/HBV+HCV, n (\%) & $0(0) / 10(83.3) / 2(16.7) / 0(0)$ & $4(4.9) / 68(84.0) / 7(8.6) / 2(2.5)$ & 0.663 \\
\hline Medical MELD score (mean \pm SD) & $15.07 \pm 5.61$ & $15.35 \pm 7.56$ & 0.821 \\
\hline \multicolumn{4}{|l|}{ Child-Pugh class } \\
\hline Class A/Class B/ Class C, n (\%) & $2(16.7) / 6(50.0) / 4(33.3)$ & $20(24.7) / 32(39.5) / 29(35.8)$ & 0.747 \\
\hline \multicolumn{4}{|l|}{ AFP level (ng/mL) } \\
\hline$\leq 400 />400, n(\%)$ & $3(25.0) / 9(75.0)$ & $75(92.6) / 6(7.4)$ & $<0.001$ \\
\hline \multicolumn{4}{|l|}{ PIVKA-II level (mAU/mL) } \\
\hline$\leq 100 />100 /$ Unknown, n (\%) & $4(36.4) / 7(63.6) / 1(8.3)$ & $63(81.8) / 14(18.2) / 4(0.5)$ & 0.001 \\
\hline PET (positive/negative), n (\%) & $8(80.0) / 2(20.0)$ & $9(13.2) / 59(86.8)$ & $<0.001$ \\
\hline Perioperative treatment (yes/no), n (\%) & $6(50.0) / 6(50.0)$ & $25(20.9) / 56(69.1)$ & 0.189 \\
\hline Milan criteria fulfilled (yes/no), n (\%) & $5(41.7) / 7(58.3)$ & $65(80.2) / 16(19.8)$ & 0.004 \\
\hline \multicolumn{4}{|l|}{ Number of tumors } \\
\hline$<3 / \geq 3, n(\%)$ & $6(50.0) / 6(50.0)$ & $60(74.1) / 21(25.9)$ & 0.086 \\
\hline \multicolumn{4}{|l|}{ Maximum tumor diameter } \\
\hline$\leq 5 \mathrm{~cm} />5 \mathrm{~cm}, \mathrm{n}(\%)$ & $9(75.0) / 3(25.0)$ & $77(95.1) / 4(4.9)$ & 0.014 \\
\hline \multicolumn{4}{|l|}{ Tumor differentiation, worst (ES grade) } \\
\hline Grade 1-2/ Grade 3-4, n (\%) & $2(16.7) / 10(83.3)$ & $45(55.6) / 36(44.4)$ & 0.012 \\
\hline Microvascular invasion (yes/no), n (\%) & $4(33.3) / 8(66.7)$ & $2(2.5) / 79(97.5)$ & $<0.001$ \\
\hline Serosal invasion (yes/no), n (\%) & $4(33.3) / 8(66.7)$ & $8(9.9) / 73(90.1)$ & 0.024 \\
\hline Intrahepatic metastasis (yes/no) & $3(30.0) / 7(70.0)$ & $10(12.3) / 71(87.7)$ & 0.132 \\
\hline \multicolumn{4}{|l|}{ Pathologic tumor stage } \\
\hline pT1/pT2-pT3 & $2(16.7) / 10(83.3)$ & $48(59.3) / 33(40.7)$ & 0.006 \\
\hline Rejection episode (yes/no), n (\%) & $2(16.7) / 10(83.3)$ & $14(17.3) / 67(82.7)$ & 0.958 \\
\hline \multicolumn{4}{|l|}{ Immunosuppression } \\
\hline Use of Basiliximab (yes/no) & $9(75.0) / 3(25.0)$ & $34(42.0) / 47$ (58.0) & 0.032 \\
\hline Tacrolimus+MMF+Steroids, n (\%) & $6(50.0)$ & $22(27.2)$ & 0.107 \\
\hline Tacrolimus+Steroids, n (\%) & $6(50.0)$ & $59(72.8)$ & \\
\hline \multicolumn{4}{|l|}{ Tacrolimus average exposure* } \\
\hline During first 1 month & $9.02 \pm 2.36$ & $9.37 \pm 2.00$ & 0.580 \\
\hline During first 3 month & $8.80 \pm 1.71$ & $8.81 \pm 1.76$ & 0.980 \\
\hline During first 6 month & $8.35 \pm 1.49$ & $8.07 \pm 1.47$ & 0.538 \\
\hline During first 1 year & $7.83 \pm 1.49$ & $7.14 \pm 1.26$ & 0.087 \\
\hline High tacrolimus exposure ${ }^{\dagger}$ (yes/no), n(\%) & $8(66.7) / 4(33.3)$ & $31(38.8) / 49(61.2)$ & 0.115 \\
\hline 1-year cumulative steroid dosage (mg), (mean $\pm S D)$ & $3620.94 \pm 538.63$ & $3788.24 \pm 749.18$ & 0.459 \\
\hline 1-year cumulative MMF dosage (g), (mean \pm SD) & $141.04 \pm 86.47$ & $231.99 \pm 120.10$ & 0.061 \\
\hline
\end{tabular}

HBV, hepatitis B virus; HCV, hepatitis C virus; MELD, model for End-Stage Liver Disease; AFP, alpha-fetoprotein; PIVKA-II, protein induced by vitamin K absence II; MMF, mycophenolate mofetil.

"Tacrolimus average exposure was defined as the area under the curve (AUC) of tacrolimus levels plotted against time courses until the time to recurrence or 1 , 3, 6 month and one year after transplantation.

${ }^{+}$High tacrolimus exposure was defined as one year average tacrolimus exposure $\geq 7.5 \mathrm{ng} / \mathrm{mL}$. 
Table 3. Comparison of the characteristics of patients with/without 3-year recurrence

\begin{tabular}{|c|c|c|c|}
\hline Variables & Recurrence group $(n=18)$ & Non- Recurrence group $(n=75)$ & $P$-value \\
\hline Age $($ mean \pm SD) & $56.6 \pm 4.23$ & $52.6 \pm 7.06$ & 0.063 \\
\hline Sex (Male/Female), n (\%) & $16(88.9) / 2(11.1)$ & $59(78.7) / 16(21.3)$ & 0.324 \\
\hline Donor type (Living/Deceased), n (\%) & $17(94.4) / 1(5.6)$ & $67(89.3) / 8(10.7)$ & 0.510 \\
\hline \multicolumn{4}{|l|}{ Etiology } \\
\hline Non-viral/HBV/HCV/ HBV + HCV, n (\%) & $0(0) / 15(83.3) / 2(11.1) / 1(5.6)$ & $4(5.3) / 63(84.0) / 7(9.3) / 1(1.3)$ & 0.530 \\
\hline Medical MELD score (mean \pm SD) & $15.07 \pm 5.61$ & $15.35 \pm 7.56$ & 0.892 \\
\hline \multicolumn{4}{|l|}{ Child-Pugh class } \\
\hline Class A/Class B/Class C, n (\%) & $4(22.2) / 8(44.4) / 6(33.3)$ & $22(23.7) / 38(40.9) / 33(35.5)$ & 0.942 \\
\hline \multicolumn{4}{|l|}{ AFP level (ng/mL) } \\
\hline$\leq 400 />400, \mathrm{n}(\%)$ & $7(38.9) / 11(61.1)$ & $71(94.7) / 4(5.3)$ & $<0.001$ \\
\hline \multicolumn{4}{|l|}{ PIVKA-II level (mAU/mL) } \\
\hline$\leq 100 />100 /$ Unknown, n (\%) & $7(38.9) / 10(55.6) / 1(5.6)$ & $60(80.0) / 11(14.7) / 4(5.3)$ & $<0.001$ \\
\hline PET (positive/negative), n (\%) & $11(68.8) / 5(31.3)$ & $6(9.7) / 56(90.3)$ & $<0.001$ \\
\hline Perioperative treatment (yes/no), n (\%) & $9(50.0) / 9(50.0)$ & $22(29.3) / 53(70.7)$ & 0.095 \\
\hline Milan criteria fulfilled (yes/ no), n (\%) & $9(50.0) / 9(50.0)$ & $61(81.3) / 14(18.7)$ & 0.006 \\
\hline \multicolumn{4}{|l|}{ Number of tumors } \\
\hline$<3 / \geq 3, \mathrm{n}(\%)$ & $9(50.0) / 9(50.0)$ & $57(76.0) / 18(21.8)$ & 0.029 \\
\hline \multicolumn{4}{|l|}{ Maximum tumor diameter } \\
\hline$\leq 5 \mathrm{~cm} />5 \mathrm{~cm}, \mathrm{n}(\%)$ & $14(77.8) / 4(22.2)$ & $72(96.0) / 3(4.0)$ & 0.008 \\
\hline \multicolumn{4}{|l|}{ Tumor differentiation, worst (ES grade) } \\
\hline Grade 1-2/ Grade 3-4, n (\%) & $2(11.1) / 6(88.9)$ & $45(60.0) / 30(40.0)$ & $<0.001$ \\
\hline Microvascular invasion (yes/no), n (\%) & $4(22.2) / 14(77.8)$ & $2(2.7) / 73(97.3)$ & 0.002 \\
\hline Serosal invasion (yes/ no), n (\%) & $8(44.4) / 10(55.6)$ & $4(5.3) / 71(94.7)$ & $<0.001$ \\
\hline Intrahepatic metastasis (yes/no), n (\%) & $5(31.3) / 11(68.8)$ & $8(10.7) / 67(89.3)$ & 0.033 \\
\hline \multicolumn{4}{|l|}{ Pathologic tumor stage } \\
\hline pT1/pT2-pT3 & $3(16.7) / 15(83.3)$ & $47(62.7) / 28(37.3)$ & $<0.001$ \\
\hline Rejection episode (yes/ no), n (\%) & $5(27.8) / 13(72.2)$ & $11(14.7) / 64(85.3)$ & 0.186 \\
\hline \multicolumn{4}{|l|}{ Immunosuppression } \\
\hline Use of Basiliximab (yes/ no) & $11(61.1) / 7(38.9)$ & $32(42.7) / 43(57.3)$ & 0.159 \\
\hline Tacrolimus + MMF + Steroids, n (\%) & $7(38.9)$ & $21(28.0)$ & 0.366 \\
\hline Tacrolimus + Steroids, n (\%) & $11(61.1)$ & $54(72.0)$ & \\
\hline \multicolumn{4}{|l|}{ Tacrolimus average exposure ${ }^{*}$} \\
\hline During first 1 month & $9.43 \pm 2.47$ & $9.30 \pm 1.94$ & 0.844 \\
\hline During first 3 month & $9.10 \pm 1.57$ & $8.73 \pm 1.79$ & 0.399 \\
\hline During first 6 month & $8.51 \pm 1.29$ & $8.01 \pm 1.50$ & 0.163 \\
\hline During first 1 year & $7.97 \pm 1.35$ & $7.05 \pm 1.24$ & 0.015 \\
\hline High tacrolimus exposure ${ }^{\dagger}$ (yes/no), n(\%) & $13(72.2) / 5(27.8)$ & $26(35.1) / 48(64.8)$ & 0.007 \\
\hline 1-year cumulative steroid dosage (mg), (mean \pm SD) & $3573.71 \pm 480.74$ & $3812.96 \pm 767.82$ & 0.211 \\
\hline 1-year cumulative MMF dosage (g), (mean \pm SD) & $177.32 \pm 124.27$ & $224.23 \pm 117.27$ & 0.374 \\
\hline
\end{tabular}

HBV, hepatitis B virus; HCV, hepatitis C virus; MELD, model for End-Stage Liver Disease; AFP, alpha-fetoprotein; PIVKA-II, protein induced by vitamin K absence II; MMF, mycophenolate mofetil

"Tacrolimus average exposure was defined as the area under the curve (AUC) of tacrolimus levels plotted against time courses until the time to recurrence or 1 , 3, 6 month and one year after transplantation.

${ }^{\dagger}$ High tacrolimus exposure was defined as one year average tacrolimus exposure $\geq 7.5 \mathrm{ng} / \mathrm{mL}$. 
period of transplant on the recurrence of aggressive nature of HCC. The impact of the immunosuppression disappeared during follow up analysis for 3-year recurrence.

In this study, exposure to immunosuppressant was analyzed using only within first postoperative year data to avoid the possible bias of a longer follow-up period of non-recurrent patients, as $66.7 \%$ of the recurrent patients were detected within one year after transplantation. The average tacrolimus exposure during first 1 , 3 , and 6 month was not significantly different between recurrence group and non-recurrence group. However, one year average tacrolimus exposure was higher in patients with recurrence. Because $38.9 \%$ of recurrence patients are detected within 6 months and the tacrolimus levels usually go to the lower end of the target range as time goes on after transplantation, the shorter follow up time of the patients who recurred early might lead to the higher calculated average tacrolimus exposure. On the other hand, despite the difference of average one year tacrolimus exposure, average tacrolimus exposure is not an independent risk factor of HCC recurrence, which is inconsistent with previous results demonstrating the effects of calcineurin inhibitors on HCC recurrence. ${ }^{7-8,21} \mathrm{Vi-}$ varelli et al. reported that the cumulative dose of cyclosporine during the first year was an independent risk factor for five-year recurrence free survival. ${ }^{4}$ The same group reported that overexposure to tacrolimus (cut-off level of $10 \mathrm{ng} / \mathrm{mL}$ ) during the first year after transplantation increased the risk of HCC recurrence. ${ }^{6}$ The suggested mechanism was that calcineurin inhibitors are involved in increased tumor growth factor- $\beta$, vascular endothelial growth factor, and the inhibition of DNA repair. ${ }^{21}$ One of the reasons we could not observe a negative effect of high tacrolimus exposure on HCC recurrence may be due to the relatively low one-year average tacrolimus exposure in both the recurrence (7.97 \pm 1.35$)$ and non-recurrence groups $(7.05 \pm 1.24)$ in this study compared with that reported in the non-recurrence group in the previous study. ${ }^{4}$ This kind of low-level tacrolimus maintenance therapy and the early tapering of steroids was possible due to most of our cases being living donor recipients with underlying hepatitis B related liver disease. ${ }^{12,14}$ Many investigators reported that early steroid withdrawal regimens reduce tumor recurrence and do not increase rejection rates. ${ }^{9}$ In our study, correlation between one-year cumulative steroid dosage and tumor recurrence was not observed because most of the patients withdrew steroids after approximately six months, and because the difference between cumulative dosages was not significant.

The Child-Pugh classes and medical MELD scores of the patients treated with basiliximab were different from the patients in the non-basiliximab group (data not shown) as expected. This selection bias could be due to the limitations of the retrospective design of this study. However, Child-Pugh classes and MELD scores were not shown as significant risk factors for HCC recurrence in the Cox-adjusted regression analysis. Induction therapy with anti-lymphocyte antibodies such as OKT-3 (anti-CD3 antibody) or anti-thymocyte globulin has been reported to increase the risk of HCC recurrence. ${ }^{22}$ Although in previous reports, basiliximab induction in combination with tacrolimus-based immunosuppressive regimens did not increase the risk of post-transplant lymphoproliferative disease, the direct impact of basiliximab on HCC recurrence has not been investigated. ${ }^{23-25}$ In a study conducted by Ramirez et al., three (18\%) recipients with HCC developed and died of metastatic HCC in the basiliximab group compared with none in the placebo group, not statistically significant. ${ }^{22}$ In a recent study based on large registry data, the use of CD25 antibody induction improved the survival rate after transplantation for HCC. However, they were not able to access the data on HCC recurrence. ${ }^{10}$ Although some research using anti-CD25 antibodies in cancer immunotherapy has been conducted, the basiliximab doses used for those purposes is different from that used in transplantation. Okita et al have demonstrated that, at low concentrations of basiliximab (under $0.06 \mu \mathrm{g} / \mathrm{mL}$ ), $\mathrm{CD} 4^{+} \mathrm{CD} 25^{\text {high }}$ regulatory $\mathrm{T}$ (T-reg) cells were selectively inhibited, allowing the tumor cells to escape from the host immune attack. ${ }^{26}$ Also, at concentrations under 0.01

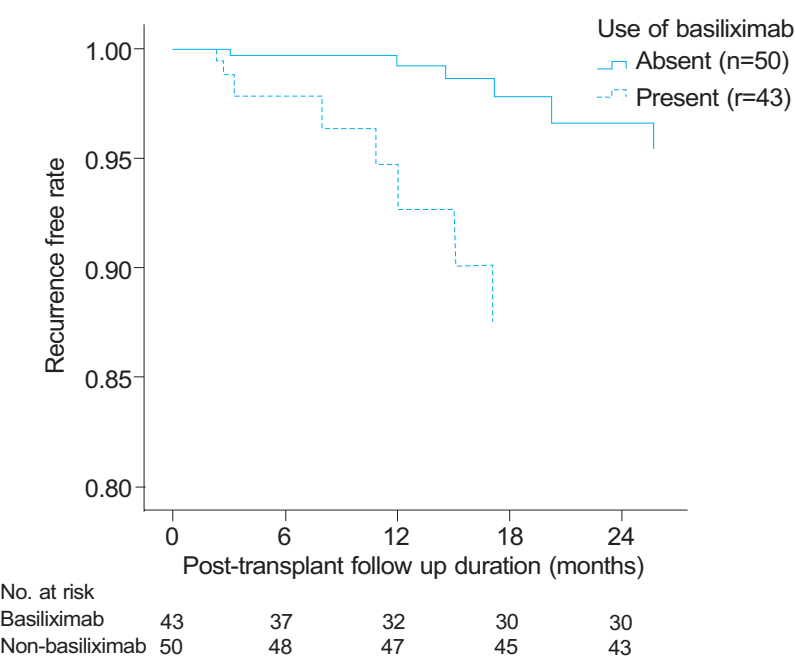

Figure 2. Recurrence-free interval curve according to induction therapy with basiliximab (Cox-adjusted model for age, gender, AFP and PIVKA-II levels, microvascular invasion, and Model for End-Stage Liver Disease score). Induction therapy with basiliximab, high AFP (>400 ng/mL) and PIVKA-II (>100 mAU/mL) levels, and microvascular invasion were significant risk factors for 1 -year recurrence $(P<0.05)$. 
$\mu \mathrm{g} / \mathrm{mL}$, basiliximab enhanced interferon-gamma production of activated peripheral blood mononuclear cells. However, at a concentration of $1 \mu \mathrm{g} / \mathrm{mL}$, an almost complete reduction of $\mathrm{CD} 4^{+} \mathrm{CD} 25^{\text {low }}$ cells to an undesirable degree, along with $\mathrm{CD} 4^{+} \mathrm{CD} 25^{\text {high }}$ cells, and the inhibition of interferon-gamma production occurs when basiliximab concentration is over $0.1 \mu \mathrm{g} / \mathrm{mL} .{ }^{26}$ After a single 20 -mg injection of basiliximab, the immediate serum concentrations of basiliximab generally ranged from 5-10 mg/L and declined over time, with a terminal half-life 13.4 days. ${ }^{27}$ Thus a relatively high dose of basiliximab (20 mg) is currently used in transplantation and results in a long-lasting, complete disappearance of all CD25+ cells, including the tumor-specific effector cytotoxic T-cells in- volved in tumor eradication. Therefore, more research is needed to confirm the correlation between induction therapy with basiliximab and HCC recurrence.

In this study, we analyzed other factors, including pathologic findings, as well as the exposure of immunosuppressive agents affecting the early recurrence of HCC. Consistent with other studies, pre-transplant MVI, AFP and PIVKA-II levels, were significantly and independently correlated with HCC recurrence. ${ }^{28-36}$ Pre-operative needle core biopsy provides exact histological information on tumors, but this procedure is not recommended due to the risk of tumor seeding. Therefore, correlation between tumor markers, i.e., serum AFP, PIVKA-II, and post-transplant histological findings is

Table 4. Univariate and multivariate Cox regression analyses of risk factors for 1-year recurrence

\begin{tabular}{|c|c|c|c|c|c|c|}
\hline \multirow{2}{*}{ Variables (n) } & \multicolumn{3}{|c|}{ Univariate analysis } & \multicolumn{3}{|c|}{ Multivariate analysis } \\
\hline & HR & $95 \% \mathrm{Cl}$ & $P$-value & HR & $95 \% \mathrm{Cl}$ & $P$-value \\
\hline Age (more than 50 years) & 6.91 & $0.89-53.57$ & 0.064 & - & - & - \\
\hline Female gender & 0.81 & $0.18-3.72$ & 0.791 & - & - & - \\
\hline Deceased donor & 0.97 & $0.13-7.50$ & 0.975 & - & - & - \\
\hline None viral/HBV/HCV/HBV+HCV & 1.39 & $0.48-3.97$ & 0.544 & - & - & - \\
\hline Child-Pugh class A/B/C & 1.15 & $0.54-2.44$ & 0.719 & - & - & - \\
\hline Medical MELD score & 0.99 & $0.90-1.09$ & 0.88 & - & - & - \\
\hline AFP level $>400 \mathrm{ng} / \mathrm{mL}$ & 24.11 & $6.47-89.91$ & $<0.001$ & 87.24 & $9.25-822.64$ & $<0.001$ \\
\hline PIVKA-II level >100 mAU/mL & 6.00 & $1.90-18.94$ & 0.002 & 11.42 & $2.04-63.83$ & 0.006 \\
\hline PET positive & 11.43 & $3.43-38.10$ & $<0.001$ & - & - & - \\
\hline Presence of preoperative treatment & 2.17 & $0.7-6.73$ & 0.18 & - & - & - \\
\hline Number of tumors $\geq 3$ & 2.77 & $0.89-8.58$ & 0.078 & - & - & - \\
\hline Maximum tumor diameter $>5 \mathrm{~cm}$ & 4.84 & $1.31-17.89$ & 0.018 & - & - & - \\
\hline Tumor differentiation G3-G4 & 5.70 & $1.25-26.00$ & 0.025 & - & - & - \\
\hline Presence of microvascular invasion & 10.57 & $3.16-35.40$ & $<0.001$ & 6.06 & $1.23-29.91$ & 0.027 \\
\hline Presence of serosal invasion & 3.9 & $1.17-12.96$ & 0.026 & - & - & - \\
\hline Presence of intrahepatic metastasis & 3.06 & $0.79-11.83$ & 0.106 & - & - & - \\
\hline Pathologic tumor stage, pT2-pT3 & 6.51 & $1.43-29.72$ & 0.016 & - & - & - \\
\hline Milan criteria unfulfilled & 5.12 & $1.62-16.13$ & 0.005 & - & - & - \\
\hline Presence of rejection episode & 0.89 & $0.20-4.08$ & 0.884 & - & - & - \\
\hline \multicolumn{7}{|l|}{ Immunosuppression } \\
\hline Use of MMF & 2.36 & $0.76-7.32$ & 0.125 & - & - & - \\
\hline Use of basiliximab & 3.87 & $1.05-14.29$ & 0.043 & 19.73 & $2.78-134.00$ & 0.003 \\
\hline High tacrolimus exposure* & 3.00 & $0.94-9.98$ & 0.073 & & & \\
\hline 1-year cumulative steroid dosage (mg) & 1.00 & $0.10-1.00$ & 0.351 & - & - & - \\
\hline 1-year cumulative MMF dosage (g) & 1.00 & $1.00-1.00$ & 0.128 & - & - & - \\
\hline
\end{tabular}

HR, hazard ratio; Cl, confidence interval; HBV, hepatitis B virus; HCV, hepatitis C virus; MELD, model for End-Stage Liver Disease; AFP, alpha-fetoprotein; PIVKA-II, protein induced by vitamin $\mathrm{K}$ absence II; MMF, mycophenolate mofetil

"High tacrolimus exposure was defined as one year average tacrolimus exposure $\geq 7.5 \mathrm{ng} / \mathrm{mL}$. 
Table 5. Univariate and multivariate Cox regression analyses of risk factors for 3-year recurrence

\begin{tabular}{|c|c|c|c|c|c|c|}
\hline \multirow[t]{2}{*}{ Variables (n) } & \multicolumn{3}{|c|}{ Univariate Analysis } & \multicolumn{3}{|c|}{ Multivariate Analysis } \\
\hline & HR & $95 \% \mathrm{Cl}$ & $P$-value & HR & $95 \% \mathrm{Cl}$ & $P$-value \\
\hline Age (more than 50 years) & 1.69 & $0.60-4.75$ & 0.317 & - & - & - \\
\hline Female gender & 0.51 & $0.12-2.22$ & 0.510 & - & - & - \\
\hline Deceased donor & 0.65 & $0.09-4.86$ & 0.671 & - & - & - \\
\hline None viral/HBV/ HCV/HBV+HCV & 1.71 & $0.77-3.79$ & 0.189 & - & - & - \\
\hline Child-Pugh class A/B/C & 1.04 & $0.57-1.92$ & 0.892 & - & - & - \\
\hline Medical MELD score & 0.99 & $0.93-1.07$ & 0.980 & - & - & - \\
\hline AFP level >400 ng/mL & 15.61 & $5.95-40.92$ & $<0.001$ & 15.99 & $4.52-57.88$ & $<0.001$ \\
\hline PIVKA-II level >100 mAU/mL & 6.50 & $2.46-17.16$ & $<0.001$ & 4.25 & $1.24-214.56$ & 0.021 \\
\hline PET positive & 11.92 & $4.12-34.53$ & $<0.001$ & 9.49 & $2.47-36.47$ & 0.001 \\
\hline Presence of preoperative treatment & 2.22 & $0.89-5.60$ & 0.090 & - & - & - \\
\hline Number of tumors $\geq 3$ & 3.22 & $1.15-9.02$ & 0.027 & - & - & - \\
\hline Maximum tumor diameter $>5 \mathrm{~cm}$ & 5.87 & $1.91-17.92$ & 0.002 & - & - & - \\
\hline Tumor differentiation G3-G4 & 9.70 & $2.23-42.17$ & 0.002 & - & - & - \\
\hline Presence of microvascular invasion & 7.13 & $2.32-21.89$ & 0.001 & & & \\
\hline Presence of serosal invasion & 6.98 & $2.74-17.76$ & $<0.001$ & - & - & - \\
\hline Presence of intrahepatic metastasis & 3.43 & $1.19-9.88$ & 0.022 & - & - & - \\
\hline Pathologic tumor stage, pT2-pT3 & 6.90 & $2.00-23.84$ & 0.002 & - & - & - \\
\hline Milan criteria unfulfilled & 3.85 & $1.53-9.71$ & 0.004 & - & - & - \\
\hline Presence of rejection episode & 1.77 & $0.63-4.96$ & 0.278 & - & - & - \\
\hline \multicolumn{7}{|l|}{ Immunosuppression } \\
\hline Use of MMF & 1.22 & $0.47-3.15$ & 0.681 & - & - & - \\
\hline Use of basiliximab & 2.12 & $0.82-5.48$ & 0.120 & & & \\
\hline High tacrolimus exposure* & 3.63 & $1.36-9.67$ & 0.008 & & & \\
\hline 1-year cumulative steroid dosage (mg) & 1.00 & $0.99-1.00$ & 0.143 & - & - & - \\
\hline 1-year cumulative MMF dosage (g) & 0.309 & $1.00-1.00$ & & - & - & - \\
\hline
\end{tabular}

HR, hazard ratio; Cl, confidence interval; HBV, hepatitis B virus; HCV, hepatitis C virus; MELD, model for End-Stage Liver Disease; AFP, alpha-fetoprotein; PIVKA-II, protein induced by vitamin K absence II; MMF, Mycophenolate mofetil

"High tacrolimus exposure was defined as one year average tacrolimus exposure $\geq 7.5 \mathrm{ng} / \mathrm{mL}$.

important to predict HCC recurrence after LT. Among several pathologic factors, MVI is one of the strongest prognostic risk factors for tumor recurrence. ${ }^{33-35}$

According to this study results, basiliximab induction therapy might have a negative impact as a strong immunosuppression on early HCC recurrence within one year. Therefore, basiliximab induction therapy should be cautious for the high risk patients of early HCC recurrence according to the guidance of pre-transplant risk factors. If the patient's pre-transplant medical condition is poor requiring basiliximab induction therapy, but the patient has HCC with poor biology, i.e., high AFP/PIVKA-II levels, a baseline immunosuppressant with a calcineurin inhibitor and a steroid must be lowered or delayed in order to avoid excessive immunosuppression. However, due to the limitations of retrospective design and relatively small sample size of our study, prospective randomized studies with large sample size are needed to confirm the impact of basiliximab induction therapy on early HCC recurrence after LT.

\section{Conflicts of Interest}

The authors have no conflicts to disclose. 


\section{REFERENCES}

1. Ferlay J, Shin HR, Bray F, Forman D, Mathers C, Parkin DM. Estimates of worldwide burden of cancer in 2008: GLOBOCAN 2008. Int J Cancer 2010;127:2893-2917.

2. Welker MW, Bechstein WO, Zeuzem S, Trojan J. Recurrent hepatocellular carcinoma after liver transplantation - an emerging clinical challenge. Transpl Int 2013;26:109-118.

3. Molmenti EP, Klintmalm GB. Liver transplantation in association with hepatocellular carcinoma: an update of the International Tumor Registry. Liver Transpl 2002;8:736-748.

4. Vivarelli M, Bellusci R, Cucchetti A, Cavrini G, De Ruvo N, Aden $A A$, et al. Low recurrence rate of hepatocellular carcinoma after liver transplantation: Better patient selection or lower immunosuppression? Transplantation 2002;74:1746-1751.

5. Fiorentino M, Altimari A, Ravaioli M, Gruppioni E, Gabusi E, Corti B, et al. Predictive value of biological markers for hepatocellular carcinoma patients treated with orthotopic liver transplantation. Clin Cancer Res 2004;10:1789-1795.

6. Parfitt JR, Marotta P, Alghamdi M, Wall W, Khakhar A, Suskin NG, et al. Recurrent hepatocellular carcinoma after transplantation: use of a pathological score on explanted livers to predict recurrence. Liver Transpl 2007;13:543-551.

7. Vivarelli M, Cucchetti A, La Barba G, Ravaioli M, Del Gaudio M, Lauro $A$, et al. Liver transplantation for hepatocellular carcinoma under calcineurin inhibitors: reassessment of risk factors for tumor recurrence. Ann Surg 2008;248:857-862.

8. Hojo M, Morimoto T, Maluccio M, Asano T, Morimoto K, Lagman M, et al. Cyclosporine induces cancer progression by a cell-autonomous mechanism. Nature 1999;397:530-534.

9. Chen ZS, He F, Zeng FJ, Jiang JP, Du DF, Liu B. Early steroid withdrawal after liver transplantation for hepatocellular carcinoma. World J Gastroenterol 2007;13:5273-5276.

10. Toso C, Merani S, Bigam DL, Shapiro AM, Kneteman NM. Sirolimusbased immunosuppression is associated with increased survival after liver transplantation for hepatocellular carcinoma. Hepatology 2010;51:1237-1243.

11. U.S. Food and Drug Administration (FDA). Rapamune highlights of prescribing information. FDA web site, <http://www.accessdata. fda.gov/drugsatfda_docs/label/2010/021083s045,021110s052lbl. pdf $>$. Accessed 2013.

12. Kwekkeboom J, Tha-In T, Tra WM, Hop W, Boor PP, Mancham S, et al. Hepatitis B immunoglobulins inhibit dendritic cells and T cells and protect against acute rejection after liver transplantation. Am J Transplant 2005;5:2393-2402

13. Yi NJ, Lee KW, Kong SY, Park KU, Lee KB, Hong G, et al. Outcome of various treatments for posttransplant hepatitis $B$ virus recurrence. World J Surg 2013;37:812-819
14. Ramirez CB, Bozdin A, Frank A, Maley W, Doria C. Optimizing use of basiliximab in liver transplantation. Transplant Research and Risk Management 2010;2:1-10.

15. Yang SH, Suh KS, Lee HW, Cho EH, Cho JY, Cho YB, et al. The role of (18)F-FDG-PET imaging for the selection of liver transplantation candidates among hepatocellular carcinoma patients. Liver Transpl 2006;12:1655-1660.

16. Ringe B, Wittekind C, Bechstein WO, Bunzendahl H, Pichlmayr R. The role of liver transplantation in hepatobiliary malignancy: a retrospective analysis of 95 patients with particular regard to tumor stage and recurrence. Ann Surg 1989;209:88-98.

17. Silva MF, Wigg AJ. Current controversies surrounding liver transplantation for hepatocellular carcinoma. J Gastroenterol Hepatol 2010;25:1217-1226.

18. Bruix J, Sherman M; Practice guidelines committee, American association for the study of liver diseases. Management of hepatocellular carcinoma. Hepatology 2005;42:1208-1236.

19. Cescon M, Ravaioli M, Grazi GL, Ercolani G, Cucchetti A, Bertuzzo $V$, et al. Prognostic factors for tumor recurrence after a 12-year, single-center experience of liver transplantations in patients with hepatocellular carcinoma. J Transplant 2010; 2010. pii:904152

20. Roayaie S, Schwartz JD, Sung MW, Emre SH, Miller CM, Gondolesi $\mathrm{GE}$, et al. Recurrence of hepatocellular carcinoma after liver transplant: patterns and prognosis. Liver Transpl 2004;10:534-540.

21. Majno P, Giostra E, Mentha G; Geneva liver cancer study. Is there a customised immunosuppressive regimen for patients transplanted with hepatocellular carcinoma? J Hepatol 2005;43:577-584.

22. Decaens T, Roudot-Thoraval F, Bresson-Hadni S, Meyer C, Gugenheim J, Durand $F$, et al. Role of immunosuppression and tumor differentiation in predicting recurrence after liver transplantation for hepatocellular carcinoma: a multicenter study of 412 patients. World J Gastroenterol 2006;12:7319-7325.

23. Ramirez CB, Doria C, di Francesco F, laria M, Kang Y, Marino IR. Basiliximab induction in adult liver transplant recipients with $93 \%$ rejection-free patient and graft survival at 24 months. Transplant Proc 2006;38:3633-3635.

24. Gruttadauria S, Vasta F, Mandala L, Cintorino D, Piazza T, Spada M, et al. Basiliximab in a triple-drug regimen with tacrolimus and steroids in liver transplantation. Transplant Proc 2005;37:2611-2613.

25. Chapman TM and Keating GM. Basiliximab: a review of its use as induction therapy in renal transplantation. Drug 2003;63:28032835.

26. Okita R, Yamaguchi Y, Ohara M, Hironaka K, Okawaki M, Nagamine I, et al. Targeting of CD4+CD25high cells while preserving CD4+CD25low cells with low-dose chimeric anti-CD25 antibody in adoptive immunotherapy of cancer. Int J Oncol 2009;34:563-572.

27. Onrust SV, Wiseman LR. Basiliximab. Drugs 1999;57:207-213.

28. Vivarelli M, Dazzi A, Zanello M, Cucchetti A, Cescon M, Ravaioli 
$M$, et al. Effect of different immunosuppressive schedules on recurrence free survival after liver transplantation for hepatocellular carcinoma. Transplantation 2010;89:227-231.

29. Montaser LM, Abbas OM, Saltah AM, Waked IA. Circulating AFP mRNA as a possible indicator of hematogenous spread of HCC cells: a possible association with HBV infection. J Egypt Natl Canc Inst 2007;19:48-60.

30. Lee KW, Suh KS. Liver transplantation for advanced hepatocellular carcinoma. J Korean Soc Transplant 2010;24:4-12.

31. Fujiki M, Takada Y, Ogura Y, Oike F, Kaido T, Teramukai S, et al. Significance of des-gamma-carboxy prothrombin in selection criteria for living donor liver transplantation for hepatocellular carcinoma. Am J Transplant 2009;9:2362-2371.

32. Shirabe K, Itoh S, Yoshizumi T, Soejima Y, Taketomi A, Aishima S, et al. The predictors of microvascular invasion in candidates for liver transplantation with hepatocellular carcinoma-with special reference to the serum levels of des-gamma-carboxy prothrombin. J
Surg Oncol 2007;95:235-240.

33. Salizzoni M, Romagnoli R, Lupo F, David E, Mirabella S, Cercutti $E$, et al. Microscopic vascular invasion detected by anti-CD34 immunohistochemistry as a predictor of recurrence of hepatocellular carcinoma after liver transplantation. Transplantation 2003;76:844848.

34. Jonas S, Bechstein WO, Steinmüller T, Herrmann M, Radke C, Berg $T$, et al. Vascular invasion and histopathologic grading determine outcome after liver transplantation for hepatocellular carcinoma in cirrhosis. Hepatology 2001;33:1080-1086.

35. Pawlik TM, Delman KA, Vauthey JN, Nagorney DM, Ng IO, Ikai I et al. Tumor size predicts vascular invasion and histologic grade: implications for selection of surgical treatment for hepatocellular carcinoma. Liver Transpl 2005;11:1086-1092.

36. Vauthey JN, Lauwers GY, Esnaola NF, Do KA, Belghiti J, Mirza N, et al. Simplified staging for hepatocellular carcinoma. J Clin Oncol 2002;20:1527-1536. 